\title{
Comportamento reológico de suspensões aquosas de cromito de lantânio
}

\section{(Rheological behaviour of lanthanum chromite aqueous suspension)}

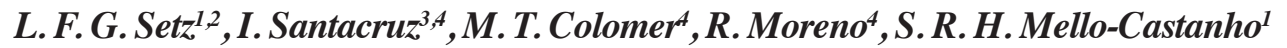 \\ ${ }^{I}$ Centro de Ciência e Tecnologia de Materiais, Instituto de Pesquisas Energéticas e Nucleares, \\ Av. Prof. Lineu Prestes 2242, Cidade Universitária, S. Paulo, SP, Brasil 05508-900 \\ ${ }^{2}$ Departamento de Engenharia de Materiais, Universidade Federal de S. Carlos, Rod. Washington Luís, km 235 \\ S. Carlos, SP, Brasil 13565-905 \\ ${ }^{3}$ Departamento de Química Inorgánica, Cristalografía y Mineralogía, Universidad de Málaga, Campus de Teatinos s/n \\ 29071 Málaga, Espanha \\ ${ }^{4}$ Instituto de Cerámica y Vidrio, Consejo Superior de Investigaciones Científicas, C/Kelsen 5, Madrid E-28049 Espanha \\ lfsetz@yahoo.com.br, isantacruz@uma.es,tcolomer@icv.csic.es,rmoreno@icv.csic.es, srmello@ipen.br
}

\begin{abstract}
Resumo
O cromito de lantânio $\left(\mathrm{LaCrO}_{3}\right)$ é o material mais estudado para a produção de interconectores para células a combustível de óxido sólido $(S O F C)$. Devido a complexidade das microestruturas e geometrias das $S O F C$, freqüentemente são necessárias, técnicas de processamento coloidal, os quais têm recebido maior atenção nos últimos anos por permitirem a obtenção de partes complexas com microestrutura controlada e reprodutíveis. Nos últimos anos, muito esforço tem sido direcionado ao processamento dos eletrólitos e eletrodos, mas aos componentes como o interconector, pouca atenção tem sido dada. Este artigo apresenta o estudo reológico e de conformação em moldes de gesso do cromito de lantânio para a produção de interconectores para SOFCs. A composição $\mathrm{La}_{0,80} \mathrm{Sr}_{0,20} \mathrm{Cr}_{0,92} \mathrm{Co}_{0,08} \mathrm{O}_{3}$, obtida por reação de combustão, foi utilizada. As suspensões aquosas foram preparadas com conteúdo de sólidos variando de 8 a 17,5\% vol. utilizando-se, poliacrilato de amônia (PAA) como polieletrólito/dispersante e hidróxido de tetrametilamônio (HTMA) como provedor de alcalinidade. A influência da concentração dos aditivos e o tempo em moinho de bolas foram estudados. Os resultados indicam que o tempo $24 \mathrm{~h}$ de homogeneização em moinho de bolas, com $3 \%$ e $1 \%$, em massa, de PAA e HTMA respectivamente, proporcionam as melhores condições para colagem em moldes de gesso, sendo possível obter peças após sinterização com densidades relativas elevadas.
\end{abstract}

Palavras-chave: cromito de lantânio, reologia, colagem em moldes porosos, interconector, SOFC.

\begin{abstract}
Lanthanum chromite $\left(\mathrm{LaCrO}_{3}\right)$ is the most studied material for SOFC's interconnectors' production. The complexity of microstructures and geometries of SOFC devices often requires the use of colloidal processing techniques, which have received increased attention in the last years for obtaining complex parts with controlled microstructure and high reliability. Much effort has been devoted to the processing of electrodes and electrolytes but the other layers, such as that of interconnecting material, have received scarce attention. This paper deals with the rheology and casting behaviour of lanthanum chromite based materials to produce interconnectors for SOFCs. A powder with the composition $\mathrm{La}_{0.80} \mathrm{Sr}_{0.20} \mathrm{Cr}_{0.92} \mathrm{Co}_{0.08} \mathrm{O}_{3}$ was obtained by combustion synthesis. Aqueous suspensions were prepared to solids loading ranging from 8 to 17.5 vol.\%, using ammonium polyacrylate (PAA) as polyelectrolyteldispersant and tetramethylammonium hydroxide (TMAH) to assure basic $\mathrm{pH}$. The influence of the additives concentrations and suspension ball milling time were studied. Suspensions prepared with $24 \mathrm{~h}$ ball milling, with $3 \mathrm{wt} \%$ and $1 \mathrm{wt} . \%$, of PAA and TMAH respectively, yielded the best conditions for successful slip casting, leading to relatively dense sintered materials.
\end{abstract}

Keywords: lanthanum chromite, rheology, slip casting, interconnector, SOFC.

\section{INTRODUÇÃO}

Atualmente, o estudo de materiais para aplicações em interconectores de células a combustível de óxido sólido (Solid Oxide Fuel Cell - SOFC), tem focalizado principalmente o cromito de lantânio $\left(\mathrm{LaCrO}_{3}\right)$, por apresentar boa performance nas condições de operação deste tipo de célula, destacando-se a resistência a ambientes redutores e oxidantes e a boa condutividade elétrica em temperaturas da ordem de $1000{ }^{\circ} \mathrm{C}$ [1]. Muitas são as geometrias utilizadas para a produção dos modelos de estudos das $S O F C$, entretanto, configurações envolvendo placas e tubos têm recebido especial atenção nos últimos anos $[2,3]$. Para estas e outras configurações com maior complexidade de forma, faz-se necessário o uso de técnicas envolvendo processamento coloidal. Neste contexto, processos de conformação como colagem em moldes porosos (slip casting), colagem em fita (tape casting), gel casting e 
screen printing têm demonstrado serem bastante eficientes na obtenção de geometrias complexas com microestruturas controladas e reprodutíveis [4, 5]. Embora a literatura apresente alguns estudos de cromito de lantânio envolvendo processamento coloidal visando a conformação por colagem em fita ou em moldes de gesso, não se encontrou estudos utilizando-se meio aquoso na produção das suspensões. Os trabalhos encontrados baseiam-se em solventes orgânicos como: etanol, tolueno, tricloroetileno, metil-etil-cetona, sozinhos ou em misturas azeotrópicas [6-10]. Neste estudo são avaliados os tempos de moagem em moinho de bolas, e os aditivos de dispersão na preparação de suspensões aquosas de cromito de lantânio, utilizando-se determinações de comportamento de fluxo por medidas reológicas.

\section{MATERIAIS E MÉTODOS}

Para a obtenção dos pós de cromito de lantânio dopado com estrôncio e cobalto utilizou-se a rota de síntese por reação de combustão, empregando-se como elementos oxidantes os nitratos dos respectivos metais: nitrato de cromo $\left(\mathrm{Cr}\left(\mathrm{NO}_{3}\right)_{3} \cdot 9 \mathrm{H}_{2} \mathrm{O}\right.$, Aldrich); nitrato de lantânio $\left(\mathrm{La}\left(\mathrm{NO}_{3}\right)_{3} \cdot 6 \mathrm{H}_{2} \mathrm{O}\right.$, Aldrich); nitrato de estrôncio $\left(\mathrm{Sr}\left(\mathrm{NO}_{3}\right)_{2}\right.$, Vetec); nitrato de cobalto $\left(\mathrm{Co}\left(\mathrm{NO}_{3}\right)_{2} \cdot 6 \mathrm{H}_{2} \mathrm{O}\right.$, Vetec) e uréia $\left(\left(\mathrm{NH}_{2}\right)_{2} \mathrm{CO}\right.$, Nuclear) como combustível, todos com grau de pureza P.A. A proporção de uréia utilizada foi estequiométrica, de acordo com trabalhos existentes [11, 12].

Baseando-se em estudos anteriores [12, 13], utilizou-se neste estudo a composição $\mathrm{La}_{0,8} \mathrm{Sr}_{0,2} \mathrm{Cr}_{0,92} \mathrm{Co}_{0,08} \mathrm{O}_{3}$. Após a síntese, as esponjas obtidas foram desagregadas em moinho atritor por duas horas utilizando-se etanol (P.A. absoluto) e esferas de nitreto de silício como elementos de moagem.

Amostras de cromito de lantânio moído foram submetidas à análise por BET visando a determinação da área de superfície específica (Monosorb, Quantachrome Instr., EUA). Após moagem, o material foi submetido a secagem em estufa a $60{ }^{\circ} \mathrm{C}$ por $24 \mathrm{~h}$. Determinou-se a distribuição de tamanho de partículas em um analisador de difração de laser (Mastersizer S, Malvern, RU) e as observações quanto à morfologia foram feitas em microscópio eletrônico de varredura com emissão de campo (MEV-EC) (Hitachi S-4700 type I, Japão).

O potencial zeta das partículas de cromito de lantânio dopado foi determinado com um zetâmetro ZetaPlus (Brookhaven Instr. Corp., EUA). As medidas de mobilidade eletroforética foram feitas em suspensões aquosas diluídas, $6,5 \cdot 10^{-3} \mathrm{~g} . \mathrm{L}^{-1}$ de $\mathrm{LaCrO}_{3}$ (água pura Milli-Q). Utilizou-se como eletrólito indiferente $\mathrm{KNO}_{3}$ com concentração fixa de $10^{-3} \mathrm{M}$. O ajuste de $\mathrm{pH}$ no intervalo de 2 a 12 foi feito adicionando-se $\mathrm{KOH} \mathrm{e} \mathrm{HNO}_{3}$. Utilizou-se também Duramax D-3005 (poliacrilato de amônia, Rohm and Haas Co., EUA) como dispersante.

As suspensões de pós de $\mathrm{LaCrO}_{3}$ foram preparadas utilizando-se água deionizada e concentração de sólidos de $42 \%$ em massa ( $10 \%$ vol.) e foram homogeneizadas em moinho de bolas utilizando esferas de alumina por tempos de até $24 \mathrm{~h}$. A influência do tempo de homogeneização fez- se comparando as medidas da viscosidade da suspensão com o tamanho das partículas. A influência da concentração de dispersante no comportamento de estabilidade das suspensões foi estudada utilizando-se o polieletrólito poliacrilato de amônio com concentrações variando de 0 a 3,5\% em massa, em relação a concentração de sólidos. A alcalinidade do meio foi obtida com adições de hidróxido de tetrametilamônio (HTMA, Sigma-Aldrich) apresentado em meio aquoso com concentração de $25 \%$ em massa, que foi considerada nos cálculos.

O comportamento reológico foi avaliado com um reômetro (Haake RS50, Thermo, Alemanha) operando nos modos de velocidade controlada (Control Rate - CR) e tensão controlada (Control Stress - CS). O sensor utilizado consistiu de um rotor duplo cone e uma placa estacionária, correspondendo a um sistema de medição do tipo Searle. O sensor é ainda protegido com uma tampa-placa para minimizar problemas de evaporação. O comportamento reológico das suspensões foi determinado pelas curvas de fluxo executadas no modo de velocidade controlada. As medidas de fluxo foram realizadas elevando-se a velocidade de cisalhamento de 0 a $1000 \mathrm{~s}^{-1}$ em 5 min, mantendo-se a $1000 \mathrm{~s}^{-1}$ for $2 \mathrm{~min}$ e retornando a $0 \mathrm{em} 5 \mathrm{~min}$. A temperatura foi mantida $25^{\circ} \mathrm{C}$ durante todo o experimento.

As suspensões analisadas no reômetro foram conformadas na forma de pequenos discos cerâmicos com diâmetro de $2 \mathrm{~cm}$ por colagem em molde de gesso. As amostras conformadas foram secas por $48 \mathrm{~h} \mathrm{em}$ temperatura ambiente e posteriormente determinou-se a densidade a verde pelo método de Arquimedes, com mercúrio como meio de imersão. Os resultados são expressos em percentagem relativa à densidade teórica $\left(\mathrm{D}_{\mathrm{t}}\right)$ do $\mathrm{La}_{0,80} \mathrm{Sr}_{0,20} \mathrm{Cr}_{0,92} \mathrm{Co}_{0,08} \mathrm{O}_{3}$ $\left(6,50 \mathrm{~g} . \mathrm{cm}^{-3}\right)$.

As peças conformadas foram sinterizadas em forno vertical a $1600{ }^{\circ} \mathrm{C} / 4 \mathrm{~h}$ de acordo com prévios trabalhos $[11,14]$.

\section{RESULTADOS E DISCUSSÃO}

Amostras do cromito de lantânio, após os ensaios de caracterização, apresentaram área de superfície específica de $10,2 \mathrm{~m}^{2} \mathrm{~g}^{-1}$ e tamanho médio de partículas de 2,08 $\mu \mathrm{m}$. Na Fig. 1a é apresentado o gráfico da curva de distribuição de tamanho de partículas que mostra uma distribuição ligeiramente heterogênea apresentando outro pico que embora com uma freqüência máxima não superior a $0,5 \%$, com tamanhos variando de 30 a $100 \mu \mathrm{m}$, possui diâmetro médio (D50) em torno de 2,08 $\mu \mathrm{m}$. Diferentemente do que se observa na micrografia obtida por MEV a partir da amostra do pó desagregado e seco (Fig. 1b), há a predominância de partículas finas, submicrométricas $(<0,3 \mu \mathrm{m})$ e com algum aglomerado, formado durante a secagem ou mesmo remanescente do processo de síntese e da desagregação. Esta diferença entre os tamanhos medidos e os observados deve-se a limitação da técnica utilizada na determinação da distribuição de tamanho de partículas que, neste caso, mediu aglomerados de partículas. Por se tratar de pós finos, 

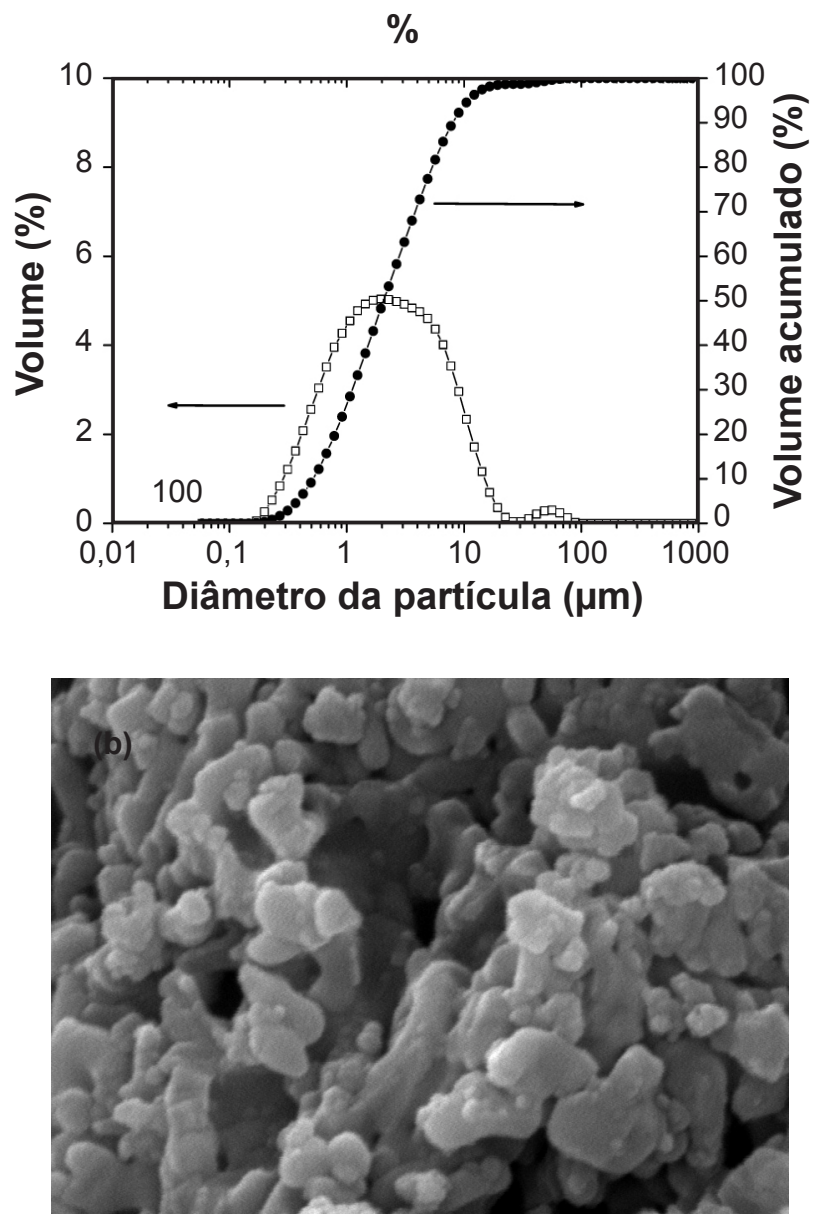

Figura 1: (a) Distribuição do tamanho de partículas do $\mathrm{LaCrO}_{3}$ e (b) micrografia MEV-EC a partir de amostra após desagregação.

[Figure 1: (a) $\mathrm{LaCrO}_{3}$ particle size distribution and (b) FESEM micrograph from disaggregated sample.]

menores que $0,3 \mu \mathrm{m}$, estes apresentam uma tendência de se aglomerar, em função da atuação de forças superficiais $[15,16]$.

Na Fig. 2 são apresentadas as curvas do potencial $\zeta$ em função do $\mathrm{pH}$ para suspensões sem e com adição de PAA (1\% em massa). O intervalo de $\mathrm{pH}$, onde as suspensões sem dispersante apresentam maiores condições de estabilidade, está entre pH 2 e 5 . Para valores de $\mathrm{pH}$ superiores a 5, suspensões de cromito de lantânio não apresentaram valores de potenciais que indicam a estabilidade para processamento. Para estas condições de preparo o ponto isoelétrico de carga ocorreu a $\mathrm{pH}_{\text {p.i.e. }}=8$. Fazendo-se o mesmo estudo, adicionando $1 \%$ m. PAA (dispersante), observa-se uma significativa mudança de comportamento das suspensões, resultado da interação do polieletrólito com as partículas do meio, principalmente para valores de $\mathrm{pH}$ superiores a 5 . Nestas condições as partículas de cromito de lantânio encontram um máximo de potencial a $\mathrm{pH}=$ 10 , podendo todavia ser trabalhada com boa estabilidade em $\mathrm{pH}=7,0$. Suspensões aquosas de cromito de lantânio modificadas com a presença de PAA apresentam ponto de isoeletricidade em $\mathrm{pH}$ entre 6 e 6,5. Os valores de potencial zeta na região ácida permanecem muito próximos nas duas condições estudadas [17].

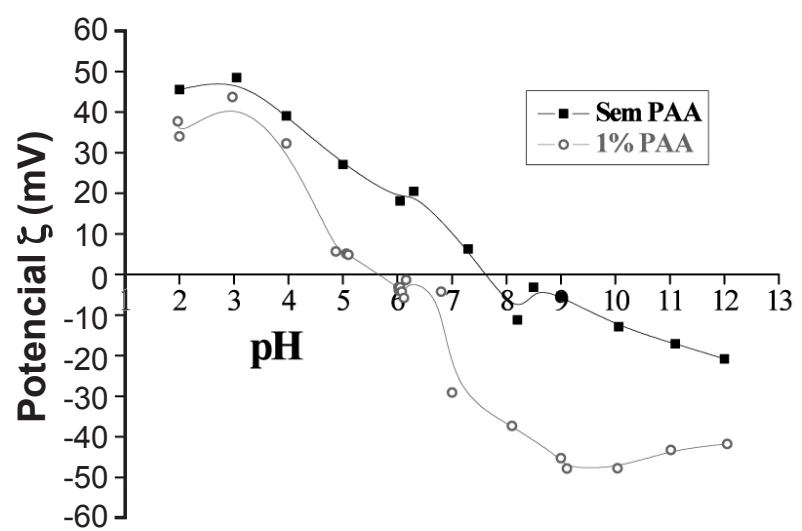

Figura 2: Variação do potencial zeta com o pH para pós de $\mathrm{LaCrO}_{3}$ sem e com adição do PAA (1\% massa).

[Figure 2: Variation of zeta potential with $\mathrm{pH}$ for $\mathrm{LaCrO}{ }_{3}$ powder with (1.0 wt.\%) and without PAA.]

Utilizando-se suspensões aquosas preparadas com $10 \%$ vol. (42\% m.) de sólidos e $1 \%$ PAA, conforme resultado apresentado na Fig. 2, estudou-se o comportamento de viscosidade dinâmica em função de $\mathrm{pH}$ nas velocidades de cisalhamento de 100,500 e $1000\left(\mathrm{~s}^{-1}\right)$. O ajuste de $\mathrm{pH}$ das suspensões foi realizado adicionando-se HTMA e, conforme ilustrado na Fig. 3, observam-se comportamentos similares da viscosidade, em função da variação de $\mathrm{pH}$, nas três velocidades. Os valores de viscosidade inicialmente a $\mathrm{pH}=7,2$, diminuem com o aumento da concentração de HTMA adicionado, ou seja, com o aumento da alcalinidade do meio, a viscosidade tende a diminuir atingindo um mínimo a pH = 9,5 (1,0\% em massa de HTMA). Como previsto na curva de estabilidade das suspensões (Fig. 2), para valores de $\mathrm{pH}>9$ a curva de potencial com a adição de PAA, tende a um valor constante. Deste modo a adição de apenas $1 \%$ m. de HTMAé suficiente para se alcançar os menores valores de viscosidade para o sistema em estudo.

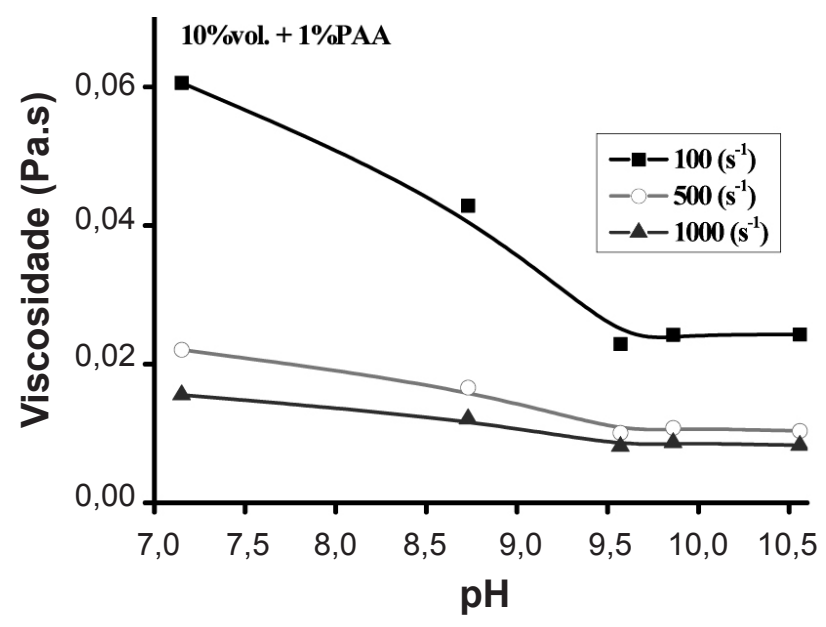

Figura 3: Variação da viscosidade da suspensão com a concentração de HTMA.

[Figure 3: Suspension viscosity versus TMAH concentration.] 
Utilizando-se a concentração de $1 \%$ m. de HTMA, estudou-se a influência da concentração de poliacrilato de amônio (PAA) no comportamento de viscosidade de suspensões de cromito de lantânio. A Fig. 4 apresenta os resultados de viscosidade dinâmica, obtidos para as suspensões em três velocidades de cisalhamento, 100, 500 e $1000\left(\mathrm{~s}^{-1}\right)$. Observa-se que, para todas as velocidades ensaiadas, ocorre um decréscimo nos valores iniciais de viscosidade com o aumento da concentração de PAA até a concentração de $2 \%$. Para maiores concentrações de PAA, os valores de viscosidade tendem a permanecerem constantes, ou seja, maiores concentrações do polieletrólito não interferem no comportamento de viscosidade da suspensão. Este fato é fortemente indicativo que nas condições de superfície deste material e, utilizando-se a concentração de sólidos em suspensão de $10 \%$ vol., o valor de $2 \%$ de PAA adicionado é o suficiente para que ocorra o recobrimento das partículas pelo polieletrólito. Ainda na Fig. 4, a acentuada redução dos valores de viscosidade na menor taxa de cisalhamento $\left(100 \mathrm{~s}^{-1}\right)$, pode ser explicada pelo fato de que, esta condição está mais próxima do repouso, apresentando uma estrutura particulada que se rompe ao ser cisalhada [4], indicando desta forma um comportamento fluidificante (pseudoplástico) $[4,18,19]$.

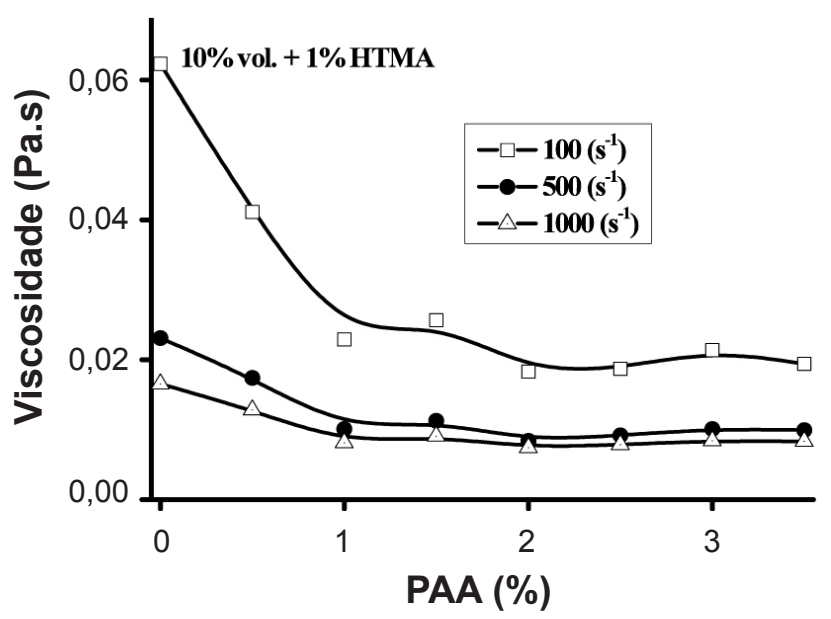

Figura 4: Variação da viscosidade em função da concentração de PAA.

[Figure 4: Viscosity variation versus PAA concentration.]

A Fig. 5 apresenta as curvas de fluxo, ou seja, a variação da tensão em função da velocidade de cisalhamento das suspensões preparadas com $10 \%$ vol. de cromito de lantânio, $1 \%$ m. de HTMA e diversas concentrações do dispersante PAA. Na medida em que se aumenta a concentração de PAA há uma redução gradual na resistência ao fluxo até a concentração de $2 \%$ m., a partir da qual as suspensões apresentam comportamento similar, confirmando o comportamento mostrado na Fig. 4. Ainda nesta figura observa-se que o aumento na concentração de PAA promove uma maior estabilidade das suspensões frente ao fluxo. Nas curvas obtidas para as suspensões sem e com $0,5 \% \mathrm{~m}$. de PAA observa-se uma diferença (histerese) entre as curvas de subida e descida (tixotropia), indicando instabilidade frente ao fluxo. Esta diferença deixa de ser observada em concentrações acima de $1 \%$ m. de PAA. A concentração que resultou nos menores valores de tensão em função da velocidade de cisalhamento, ou seja, menor viscosidade e, portanto, melhor condição de empacotamento das partículas, foi de $2 \% \mathrm{~m}$.

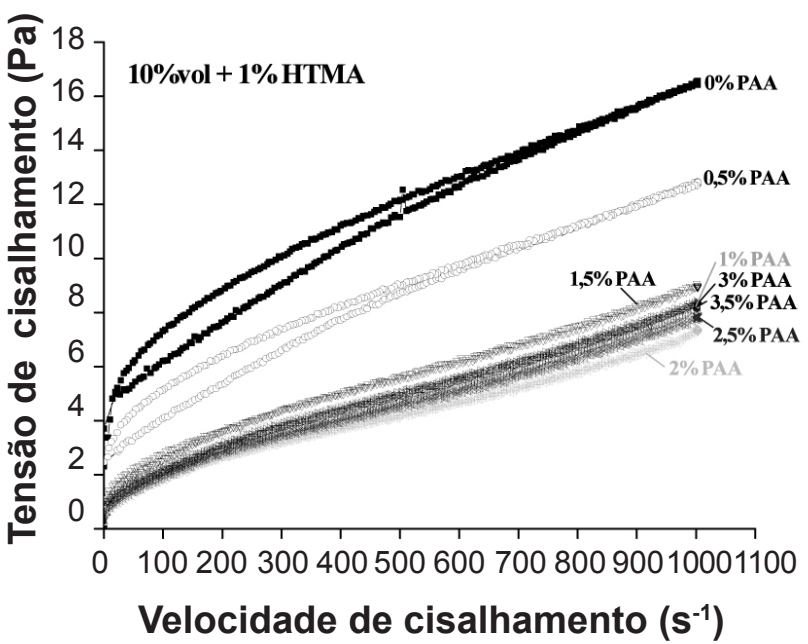

Figura 5: Curva de fluxo para diversas concentrações de PAA. [Figure 5: Flow curves of suspensions prepared with different PAA concentrations.]

Determinadas as condições de $\mathrm{pH}$ do meio, obtido com $1 \%$ de HTMA, e da concentração do dispersante PAA (2\%), estudou-se a influência do tempo de homogeneização, em moinho de bolas, no comportamento das suspensões. A Fig. 6 apresenta este comportamento, onde se observa que a redução no tamanho das partículas do meio em função do tempo de moagem, é acompanhada de um aumento na viscosidade dinâmica, medida a velocidade de $10 \mathrm{~s}^{-1}$, até o tempo de $24 \mathrm{~h}$. Este comportamento indica um aumento na área de superfície específica do pó adicionado conforme se aumenta o tempo de moagem e, desta maneira, há um aumento na quantidade de partículas no sistema, que conduzem a um aumento na resistência ao fluxo. As viscosidades dinâmicas na velocidade de cisalhamento de $10 \mathrm{~s}^{-1}$ são consideradas, pois geralmente, no processo de colagem em moldes de gesso, esta é a velocidade alcançada. Os valores de viscosidade, nesta velocidade, são elevados por estarem mais próximas da condição de repouso [4]. Tempos de moagem superiores a $24 \mathrm{~h}$ não provocaram mudanças nas propriedades das suspensões e, portanto, $24 \mathrm{~h}$ em moinho de bolas foram considerados neste estudo.

Após submeter a suspensão ao tempo de homogeneização de $24 \mathrm{~h}$ e verificar um aumento no valor de sua viscosidade dinâmica, fez-se o estudo do reajuste na concentração de dispersante. A Fig. 7, contendo curvas de fluxo tensão por velocidade de cisalhamento apresenta este estudo, onde, com um incremento de $1 \%$ de PAA, totalizando um total de $3 \%$ m., a suspensão adquire um valor mínimo na resistência ao fluxo. A partir disso, aumentos na concentração de PAA não modificam o comportamento da suspensão, indicando que, 


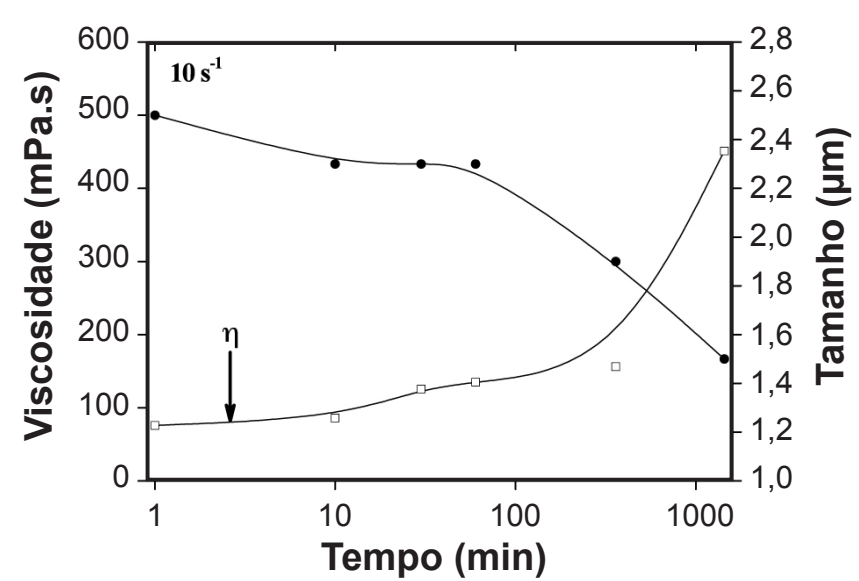

Figura 6: Variação do tamanho médio de partículas com o tempo de moagem, comparada com a variação da viscosidade.

[Figure 6: Average particle size variation versus milling time, compared with viscosity variation.]

as partículas estão plenamente recobertas, já nesta condição.

O reajuste da concentração de HTMA não foi necessário, uma vez que, após $24 \mathrm{~h}$ de moagem, não ocorreu significativa alteração no valor de $\mathrm{pH}$ da suspensão $(\mathrm{pH} \sim 9,0)$.

Definidas as concentrações do dispersante (3\% de PAA) e da base (1\% de HTMA), necessárias à estabilização do cromito de lantânio em água, estudou-se então o aumento da concentração de sólidos na suspensão, apresentada na Fig. 8. A curva, determinada para diversas concentrações de sólidos, foi obtida com os valores da viscosidade medida na velocidade de cisalhamento de $10 \mathrm{~s}^{-1}$. Nela se observa um aumento na viscosidade dinâmica com o aumento na concentração, até um máximo de aproximadamente $20 \%$ vol., isso significa que, para o $\mathrm{LaCrO}_{3}$ dopado com $\mathrm{Sr}$ e Co, preparado nesta condição, o aumento na concentração de sólidos até a condição de suspensão, ou seja, fluida e capaz de ser vertida, chegou a um valor máximo. Nestas condições e para este material, a partir de $\sim 17 \%$ vol. a

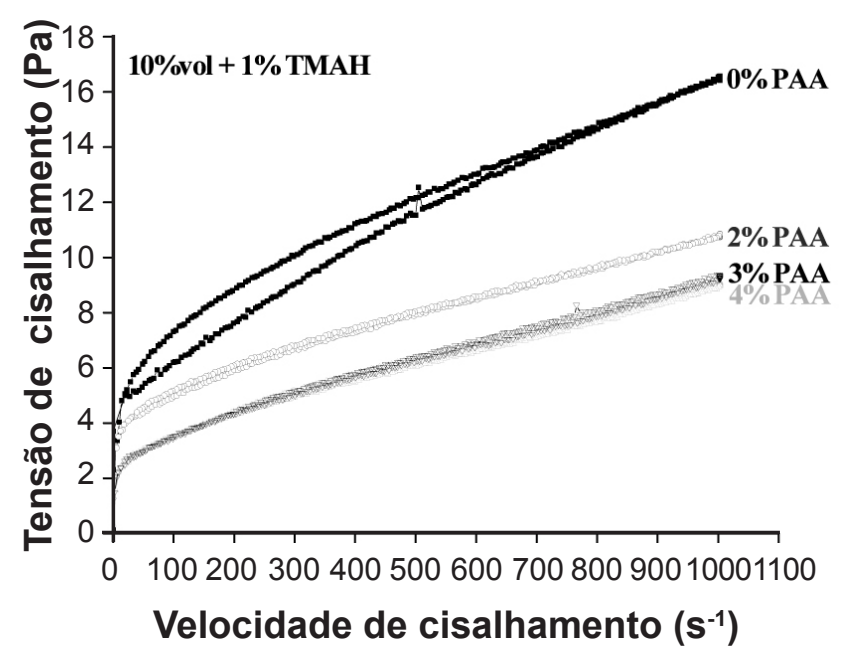

Figura 7: Curvas de fluxo para suspensões reajustadas com PAA. [Figure 7: Flow curves for PAA readjusted suspensions.]

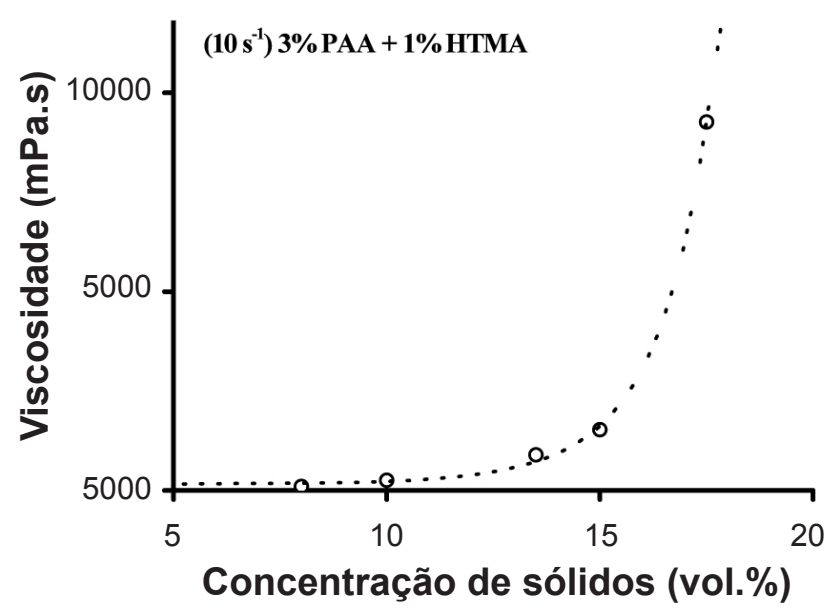

Figura 8: Variação da viscosidade com a concentração de sólidos. [Figure 8: Viscosity variation versus volume fraction of solids.]

suspensão apresentou aspecto pastoso, com uma viscosidade de $~ 9000 \mathrm{mPa} . \mathrm{s}$, muito superior ao valor de viscosidade desejado (2000 mPa.s) [5] para conformação em moldes porosos, sendo portanto inadequada para colagem em gesso. A limitação no aumento da concentração de sólidos, nas suspensões aquosas de cromito de lantânio dopado com estrôncio e cobalto, é atribuída à sua solubilidade em água [17].

A análise reológica das suspensões com diferentes conteúdos de sólidos é apresentada na Tabela I. Nesta tabela se observa que o aumento da concentração promoveu um incremento no valor de tixotropia e como esperado, ocorreu um aumento no valor da viscosidade dinâmica. Os valores de tixotropia negativos são atribuídos ao limite de erro do equipamento (5\%), e apresentam elevados valores, pois as medidas foram obtidas considerando-se toda a extensão estudada ( 0 a $\left.1000 \mathrm{~s}^{-1}\right)$, porém, os valores apresentaram uma evolução linear. Os valores das densidades relativas a verde das peças conformadas também aumentaram com o aumento na concentração de sólidos.

As densidades relativas das peças conformadas em moldes de gesso, com diferentes conteúdos de sólido e sinterizadas a $1600{ }^{\circ} \mathrm{C} / 4 \mathrm{~h}$, são apresentadas também na Tabela I. Todas as peças coladas apresentam-se bastante densas, com valores acima de $98 \% \mathrm{D}_{\mathrm{t}}$.

A Fig. 9 mostra as micrografias de fratura das peças sinterizadas $\left(1600{ }^{\circ} \mathrm{C} / 4 \mathrm{~h}\right)$ contendo 15 e $17,5 \%$ vol. de sólidos. Nas amostras é possível observar porosidade intergranular e intragranular, condizentes com os valores obtidos das densidades relativas, e aspecto de fratura intragranular e intergranular, semelhante aos reportados $[6,20,21]$. Observa-se também um predomínio de ruptura intergranular em menores concentrações de sólidos (Fig. 9a) e de fratura intragranular em maiores concentrações (Fig. 9b). Desta maneira, o processo de conformação em moldes porosos (slip casting) proporcionou, após a sinterização, peças com elevados valores de densidades, adequadas para utilização do cromito de lantânio dopado com estrôncio e cobalto como interconector. O parâmetro densidade é 
Tabela I - Parâmetros reológicos das suspensões contendo diferentes conteúdos de sólidos, homogeneizados por $24 \mathrm{~h}$, com $3 \% \mathrm{~m}$. de PAA e $1 \% \mathrm{~m}$. de HTMA.

[Table I-Rheological parameters of suspensions with various solids content, homogenized for $24 \mathrm{~h}$, with PAA 3 wt.\% and TMAH 1 wt.\%.]

\begin{tabular}{cccccc}
\hline $\begin{array}{c}\text { Concentração } \\
\text { de sólidos } \\
(\% \text { vol. })\end{array}$ & $\begin{array}{c}\text { Concentração } \\
\text { de sólidos } \\
(\% \text { m. })\end{array}$ & $\begin{array}{c}\text { Tixotropia } \\
\left(\mathrm{Pa} .^{-1}\right)\end{array}$ & $\begin{array}{c}\text { Viscosidade } \\
\mathrm{a} 10 \mathrm{~s}^{-1} \\
(\mathrm{mPa} . \mathrm{s})\end{array}$ & $\begin{array}{c}\text { Densidade a } \\
\text { verde } \\
(\% \mathrm{D})\end{array}$ & $\begin{array}{c}\text { Densidade } \\
\text { sinterizada } \\
\left(\% \mathrm{D}_{\mathrm{t}}\right)\end{array}$ \\
\hline 8,0 & 36,1 & $-47,7$ & 99,9 & $33,6 \pm 0,2$ & $98,8 \pm 0,3$ \\
10,0 & 41,9 & $-154,5$ & 258,5 & $34,2 \pm 0,2$ & $98,5 \pm 0,3$ \\
13,5 & 50,4 & $-821,3$ & 892,6 & $30,6 \pm 0,2$ & $98,8 \pm 0,3$ \\
15,0 & 53,4 & $-1145,0$ & 1527,0 & $36,9 \pm 0,2$ & $98,9 \pm 0,3$ \\
17,5 & 58,0 & $-2642,0$ & 9269,0 & $36,2 \pm 0,2$ & $98,5 \pm 0,3$ \\
\hline
\end{tabular}
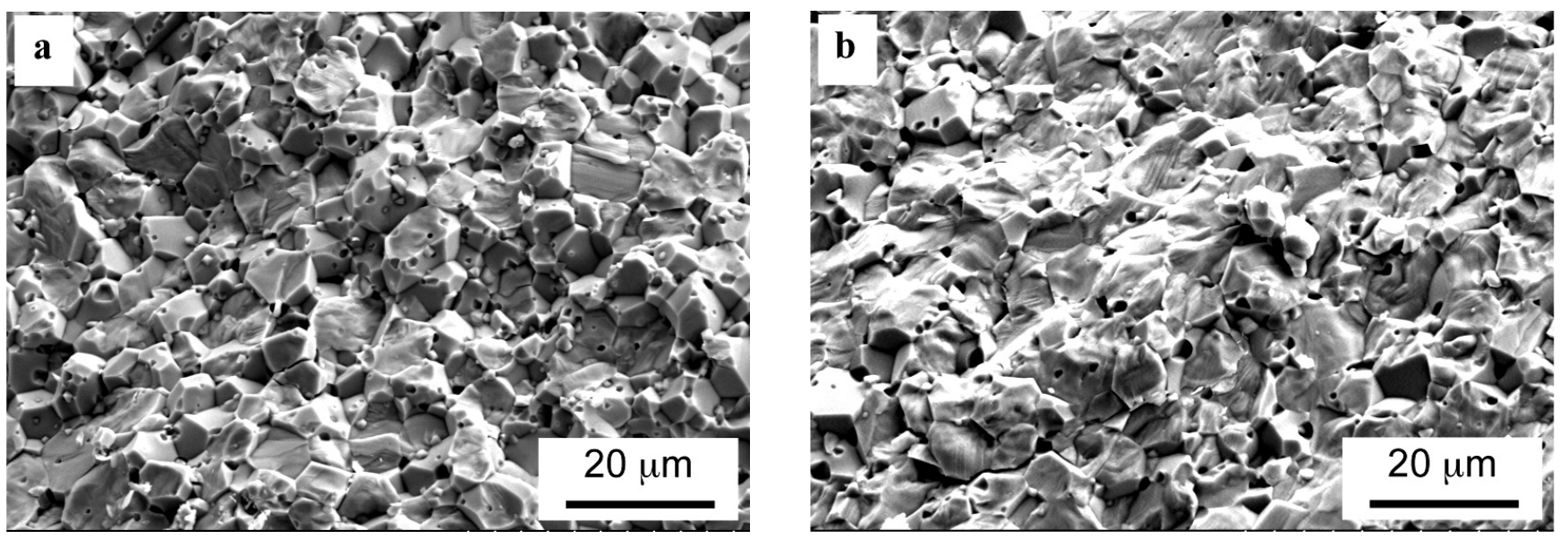

Figura 9: Micrografia MEV-EC das superfícies de fratura das peças sinterizadas, coladas em moldes de gesso através de suspensões contendo (a) 15 e (b) $17,5 \%$ vol. de sólidos.

[Figure 9: FEG-SEM micrographs of the fresh fracture surfaces for the sintered specimens cast from suspensions with (a) 15 and (b) 17.5 vol.\% solids loading.]

importante nos interconectores das SOFCs uma vez que estes componentes necessitam ser densos para evitar a mistura dos gases reagentes, o hidrogênio e o oxigênio, durante a operação da célula a combustível.

\section{CONCLUSÕES}

É possível a preparação de suspensões aquosas de cromito de lantânio para colagem em moldes de gesso. As melhores condições reológicas foram obtidas dispersando as suspensões com poliacrilato de amônio e hidróxido de tetrametilamônio, que promovem uma estabilização eletrostérica em um pH básico capaz de alinhar as cadeias poliméricas.Concentrações em massade $3 \%$ de poliacrilato de amônio e $1 \%$ de hidróxido de tetrametilamônio promoveram os melhores resultados após $24 \mathrm{~h}$ de homogeneização em moinho de bolas. Os valores das densidades relativas em verde $\left(\sim 30 \% D_{\mathrm{t}}\right)$ levaram a obtenção de materiais sinterizados com densidades relativas altas $\left(\sim 98 \% \mathrm{D}_{\mathrm{t}}\right)$ e com alguma porosidade remanescente intra e intergranular que, entretanto, podem ser melhoradas. Os resultados apresentados neste o trabalho, todavia, mostraram-se bastante adequados à produção de interconectores, uma vez que não existem na literatura trabalhos utilizando-se de meio aquoso no processamento coloidal do cromito de lantânio.

\section{AGRADECIMENTOS}

Ao MCT - Finep - CNPq (Proc. $n^{\circ} 142855 / 2005-7$ ) e à CAPES (Proc. $\mathrm{n}^{\circ}$ 4710-06-1) pelas bolsas de doutorado e doutorado sanduíche, respectivamente, e à FAPESP (Proc. 2009/54851-6) pela bolsa de Pós-Doutorado de L. F. G. Setz. Agradecem também ao Ministério de Ciência e Inovação da Espanha - MICINN, pelo aporte financeiro (MAT200914369-C02-01) e pela concessão da bolsa Ramón y Cajal (RYC-2008-03523) à Dra. M. I. Santacruz.

\section{REFERÊNCIAS}

[1] N. Q. Minh, J. Am. Ceram. Soc. 76 (1993) 563.

[2] H. Yoshida, H. Yakabe, K. Ogasawara, T. Sakurai, J. Power Sources 157 (2006) 775.

[3] N. M. Sammes, Y. Du, Int. J. Appl. Ceram. Technol. 4 (2007) 89. 
[4] R. Moreno, "Reología de Suspensiones Cerámicas", Consejo Superior de Investigaciones Científicas, Madrid, Espanha (2005).

[5] J. S. Reed, "Principles of Ceramics Processing", John Wiley \& Sons, Inc., New York, EUA (1995).

[6] M. W. Murphy, T. R. Armstrong, P. A. Smith, J. Am. Ceram. Soc. 80 (1997) 165.

[7] N. M. Sammes, R. Ratnaraj, J. Mater. Sci. Lett. 13 (1994) 678.

[8] N. M. Sammes, R. Ratnaraj, J. Mater. Sci. Lett. 13 (1994) 1664.

[9] N. M. Sammes, C. E. Hatchwell, Mater. Lett. 32 (1997) 339.

[10] L. F. G. Setz, I. Santacruz, M. T. Colomer, S. R. H. Mello-Castanho, R. Moreno, J. Eur. Ceram. Soc. 30 (2010) 2897.

[11] L. F. G. Setz, H. P. S. Corrêa, C. Yamagata, S. R. H. Mello-Castanho, "Advances in Solid Oxide Fuel Cells III: Ceram. Eng. Sci. Proc.”, John Wiley \& Sons, Westerville, EUA (2007) 239.

[12] L. F. G. Setz, S. R. H. Mello-Castanho, R. Moreno, M. T. Colomer, Int. J. Appl. Ceram. Technol. 6 (2009) 626.
[13] L. F. G. Setz, "Processamento Coloidal de Cromito de Lantânio", Tese Dr., Instituto de Pesquisas Energéticas e Nucleares, USP, S. Paulo, Brasil (2009).

[14] L. F. G. Setz, H. P. S. Corrêa, C. O. Paiva-Santos, S. R. H. Mello-Castanho, Mater. Sci. Forum 530-531 (2006) 671. [15] J. N. Israelachvili, "Intermolecular and Surface Forces", $2^{\text {nd }}$ Ed., Academic Press, Oxford, UK (1992).

[16] D. J. Shaw, "Introduction to Colloid and Surface Chemistry", $4^{\text {th }}$ Ed., Butterworth-Heinemann, Oxford, UK (1992).

[17] L. F. G. Setz, S. R. H. Mello-Castanho, M. T. Colomer, R. Moreno, Solid State Ionics 180 (2009) 71.

[18] I. R. Oliveira, A. R. Studart, R. G. Pileggi, V. C. Pandolfelli, "Dispersão e Empacotamento de Partículas - Princípios e Aplicações em Processamento Cerâmico", Fazendo Arte Editorial, S. Paulo, Brasil (2000).

[19] J. F. Steffe, "Rheological Methods in Food Process Engineering", $2^{\text {nd }}$ Ed., Freeman Press, East Lansing, EUA (1996).

[20] S. W. Paulik, S. Baskaran, T. R. Armstrong, J. Mater. Sci. 33 (1998) 2397.

[21] Z. Zhong, Solid State Ionics 177 (2006) 757. (Rec. 13/08/2010, Rev.09/09/2010, Ac. 14/10/2010) 